Revue internationale P.M.E.

Économie et gestion de la petite et moyenne entreprise

\title{
Construire son Business Plan, Catherine Léger-Jarniou et Georges Kalousis Paris, Dunod, 2006, 241 p.
}

\section{André Cyr}

Volume 21, numéro 1, 2008

URI : https://id.erudit.org/iderudit/038006ar

DOI : https://doi.org/10.7202/038006ar

Aller au sommaire du numéro

Éditeur(s)

Presses de l'Université du Québec

ISSN

0776-5436 (imprimé)

1918-9699 (numérique)

Découvrir la revue

Citer ce compte rendu

Cyr, A. (2008). Compte rendu de [Construire son Business Plan, Catherine

Léger-Jarniou et Georges Kalousis Paris, Dunod, 2006, 241 p.] Revue

internationale P.M.E., 21(1), 143-144. https://doi.org/10.7202/038006ar d'utilisation que vous pouvez consulter en ligne. 


\section{Comptes rendus}

\section{CONSTRUIRE SON BUSINESS PLAN Catherine Léger-Jarniou et Georges Kalousis Paris, Dunod, 2006, 241 p.}

Construire son Business Plan est un guide pratique pour chaque entrepreneur qui vise créer un plan d'affaires complet et réfléchi. Basé sur l'expérience pratique des auteurs en consultation auprès de jeunes entreprises, ce livre aide à démystifier le «business plan» et à décortiquer le processus de sa création en étapes faciles à suivre. Rédigé dans un langage simple et compréhensible, l'ouvrage peut s'adresser à un public très large, même s'il introduit des notions clés en matière d'économie, de finance et de management (par exemple, le modèle de Porter, le coût de revient, la chaîne de valeur, la matrice BCG, etc.). Ces renvois théoriques sont clarifiés dans un lexique séparé à la fin du livre et sont traités d'un point de vue purement pratique dans le corps. Construire son Business Plan s'adresse directement aux entrepreneurs et donne des conseils, des astuces et des ressources tirés de l'expérience sur le terrain des auteurs. Même si les références et les informations juridiques, comptables et financières sont valides pour la France et l'Union européenne, le livre conserve sa qualité de guide, étant donné que le lecteur peut facilement trouver des équivalents québécois. L'usage fréquent de termes anglo-saxons (Business Plan, check-list, etc.) là où des mots français existent est parfois agaçant et diminue la qualité générale du texte.

Construire son Business Plan comprend deux parties, chacune composée de deux chapitres. La première partie traite du plan d'affaires comme tel et la seconde, des étapes du processus de sa construction.

Dans le premier chapitre, les auteurs discutent la nature du plan d'affaires, de sa raison d'être, de ses objectifs et de ses différents publics (partenaires internes et externes, banquiers, capital-risqueurs). Des conseils sont donnés sur la rédaction et la présentation en fonction du but poursuivi: démarrage de l'entreprise, recherche de financement ou suivi et développement stratégique. La double nature du business plan (document de travail et outil de communication) est aussi discutée. Enfin, un survol du contenu des différentes composantes, ainsi que quelques modèles types de plan d'affaires sont présentés au lecteur pour l'aider à produire un document synthétique et prospectif, capable de susciter l'intérêt.

Après avoir analysé l'utilité du plan d'affaires et les manières de le vendre et de le défendre, dans le deuxième chapitre, les auteurs s'attardent sur les renseignements nécessaires à sa construction. La nécessité de rassembler de nombreuses données managériales et environnementales pour bien définir 
son projet, délimiter le périmètre de son activité et prévoir le mode de fonctionnement de son organisation est mise en évidence. Une «check-list» des données indispensables est fournie et son utilité est discutée. Le moment opportun du démarrage et la prise en compte des ressources et des dépenses en fonction de la nature du projet (industriel, commercial ou technologique) sont aussi analysés dans ce chapitre.

La deuxième partie du livre est consacrée à la construction du plan d'affaires : la partie descriptive (chapitre 3 ) et sa traduction en termes financiers (chapitre 4). Dans le chapitre sur l'élaboration du texte, à part d'approfondir les éléments constitutifs du business plan (historique, description du marché et de la concurrence, technologie et moyens de protection, etc.), les auteurs portent une attention particulière sur la cohérence entre ces éléments et leur traduction financière: les ambitions de l'entrepreneur eu égard aux moyens dont il dispose ou peut mobiliser, la taille du marché visé par rapport à ces mêmes moyens, etc. Aussi, différents outils théoriques (modèle de Porter, matrices BCG et ADL, etc.) et pratiques (tableaux détaillés des étapes et des délais des procédures) sont mis à disposition du lecteur pour l'aider à cristalliser ses choix stratégiques et à les expliquer.

Le dernier chapitre du livre met l'accent sur la construction des tableaux financiers: plan de financement initial, bilan prévisionnel, états financiers prévisionnels, fonds de roulement, seuil de rentabilité, etc. Cette partie est illustrée avec le cas d'une entreprise et fait, en même temps, la présentation d'un outil informatique facilitant le montage des tableaux financiers. Une rédaction simultanée de la partie texte et de la traduction financière est suggérée afin de déceler toute incohérence possible (sous- ou surévaluation du projet).

En conclusion, les auteurs rappellent que la transformation d'une idée en projet d'entreprise est un processus laborieux qui se traduit concrètement par le plan d'affaires. Ils soulignent le caractère itératif de ce processus et répètent que le business plan est à la fois un document de travail et un outil de communication qui sert à convaincre les différents partenaires, à «vendre » le projet, à susciter l'intérêt.

Construire son Business Plan est une bonne source d'information et un guide pratique sur la manière de rédiger un plan d'affaires pour le grand public. Ce livre accompagne l'entrepreneur tout au long du processus, à partir de la conception jusqu'à la présentation en fournissant des outils, des mises en garde et des exemples pratiques. Même si les informations et les ressources juridiques, comptables et financières concernent la France et l'Union européenne, cet ouvrage peut être très utile à cause du regard complexe qu'il porte sur les différents aspects du démarrage de l'entreprise et leurs interrelations.

André Cyr, Ph.D.

Université du Québec à Trois-Rivières

Revue internationale P.M.E., vol. 21, $\mathrm{n}^{\circ}$ 1, 2008 